\title{
RADIATIVE TRANSFER EFFECTS DURING PHOTOHEATING OF THE INTERGALACTIC MEDIUM
}

\author{
Tom Abel ${ }^{1,2}$ and Martin G. HaEhnelT ${ }^{2}$ \\ Draft version January 31, 2018
}

\begin{abstract}
The thermal history of the intergalactic medium (IGM) after reionization is to a large extent determined by photoheating. Here we demonstrate that calculations of the photoheating rate which neglect radiative transfer effects substantially underestimate the energy input during and after reionization. The neglect of radiative transfer effects results in temperatures of the IGM which are too low by a factor of two after He II reionization. We briefly discuss implications for the absorption properties of the IGM and the distribution of baryons in shallow potential wells.
\end{abstract}

Subject headings: quasars: absorption lines - galaxies: formation - cosmology: theory

\section{INTRODUCTION}

The absence of a Gunn-Peterson trough (Gunn \& Peterson 1965, Scheuer 1965) in the spectra of high-redshift objects is solid evidence that the Universe has been reionized before $z \sim 5$. Hydrodynamical simulations have shown convincingly that at redshifts $z \sim 3$ most baryons are still contained in a photoionized IGM which is responsible for the Ly $\alpha$ forest in the absorption spectra of high-redshift QSO's (Cen et al. 1994, Petitjean, Mücket \& Kates 1995; Zhang, Anninos \& Norman 1995; Hernquist et al. 1996). However, the detailed distribution of the baryonic component as well as the absorption properties of the IGM will depend on the thermal history of the IGM and thus the heat input due to photoheating (Efstathiou 1992, MiraldaEscude \& Rees 1994, Navarro \& Steinmetz 1997, Haehnelt \& Steinmetz 1998, Bryan et al. 1999; Theuns et al. 1999). Most numerical simulations so far adopted the optically thin limit when calculating photoheating rates. This is, however, a bad approximation during reionization when the optical depth is large. As we will argue later in the case of helium it is also a bad approximation after He II reionization (see also Giroux \& Shapiro 1996). Here we investigate radiative transfer effects during and after reionization and discuss implications for the thermal history and absorption properties of the IGM and the distribution of baryons in shallow potential wells. We assume an Einstein-de-Sitter Universe and a Hubble constant of $50 \mathrm{~km} \mathrm{~s}^{-1} \mathrm{Mpc}^{-1}$.

\section{PHOTOHEATING}

\subsection{Optical thin vs optical thick}

In the optically thin limit the mean excess energy of ionizing photons is given by

$$
\left\langle E_{\mathrm{ph}}^{\mathrm{thin}}\right\rangle=\frac{\int_{\nu_{\mathrm{th}}}^{\infty} \frac{J(\nu)}{h \nu} \sigma(\nu)\left(h \nu-h \nu_{t h}\right) d \nu}{\int_{\nu_{\mathrm{th}}}^{\infty} \frac{J(\nu)}{h \nu} d \nu}
$$

where $J(\nu)$ is the flux of ionizing photons $\sigma(\nu)$ is the crossection for ionization, $\nu_{t h}$ the threshold energy for ionization, and $h$ Planck's constant. For a powerlaw spectrum $J(\nu)=J_{0}\left(\nu / \nu_{t h}\right)^{-\alpha}$ and a powerlaw approximation $\left(\propto \nu^{-3}\right)$ for the crossection one finds $\left\langle E_{\mathrm{ph}}^{\mathrm{thin}}\right\rangle \approx$ $h \nu_{\mathrm{th}} /(\alpha+2) \equiv E_{\mathrm{th}} /(\alpha+2)$. For spectral indices of interest $\alpha>1$ these excess energies are small fractions of the threshold energy $E_{\mathrm{th}}$. This is because the integral in equation (1) is strongly weighted towards the threshold energy. In the optical thick limit where every ionizing photon emitted is absorbed the mean excess energy is typically much larger than $\left\langle E_{\mathrm{ph}}^{\mathrm{thin}}\right\rangle$ of equation $(1)$. For the powerlaw spectrum discussed above $\left\langle E_{\mathrm{ph}}^{\text {thick }}\right\rangle \approx h \nu_{\mathrm{th}} /(\alpha-1)$.

\subsection{Energy input during reionization}

A non-equilibrium multi-frequency 1-D radiative transfer code is used to study the thermal structure of R-type ionization fronts surrounding a point source in spherical symmetry (Abel, Meiksin, \& Norman 1999). The code evolves the cooling/heating and chemistry model for primordial gas of Abel et al. (1997) with the methods discussed by Anninos et al. (1997). It solves the static radiative transfer equation in an expanding universe and explicitly conserves photons (Abel, Norman and Madau 1999).

Figure 1 shows radial temperature profiles of two different ionization fronts around a luminous QSO (photon emission rate $\left.N_{\mathrm{ph}}=3 \times 10^{56} \mathrm{~s}^{-1}, \epsilon(\nu)=\epsilon_{0}\left(\nu / \nu_{0}\right)^{-1.8}\right)$ at $z=6$ and $z=4$, respectively. In both cases the front evolves into a homogeneous IGM of primordial composition and mean baryonic density $\left(\Omega_{\text {bar }} h^{2}=0.02\right)$. In the first case the IGM is assumed to be completely neutral and hydrogen and helium reionization occur almost simultaneously. In the second case the gas is assumed to be predominantly in the form of HII and HeII due to a soft UV background already present when the QSO switches on. For both models the ionization front is shown after $10^{7}$ and $10^{8} \mathrm{yr}$.

In the first model the ionization front is very sharp due to the small mean free path of hydrogen ionizing photons and the temperature behind the reionization front rises to $50000 \mathrm{~K}$. This corresponds to $\left\langle E_{\mathrm{ph}}\right\rangle \sim 0.85 \times E_{\mathrm{th}}$. This is much larger than in the optical thin approximation,

${ }^{1}$ Laboratory for Computational Astrophysics, NCSA, University of Illinois at Urbana/Champaign, 405 N. Mathews Ave., Urbana, IL 61801.

${ }^{2}$ Max-Planck-Institut für Astrophysik, Karl-Schwarzschild-Strasse 1, 85748 Garching, Germany 
$\left\langle E_{\mathrm{ph}}^{\mathrm{thin}}\right\rangle=0.26 E_{\mathrm{th}}$, and approaches the optical thick limit, $\left\langle E_{\mathrm{ph}}^{\text {thick }}\right\rangle=1.25 E_{\mathrm{th}}$, for the adopted power-law spectrum with $\alpha=1.8$. The difference is mainly due to line-cooling of the neutral component in the ionization front. This becomes more apparent at later times when the ionization front has slowed down. Hydrogen line cooling of the neutral component during the passage of the ionization front becomes then more effective and the temperature falls to $28000 \mathrm{~K}$ (Miralda-Escude \& Rees 1994). This is still nearly a factor two larger than the value of $15000 \mathrm{~K}$ in the optical thin limit (e.g. Haehnelt \& Steinmetz 1998).

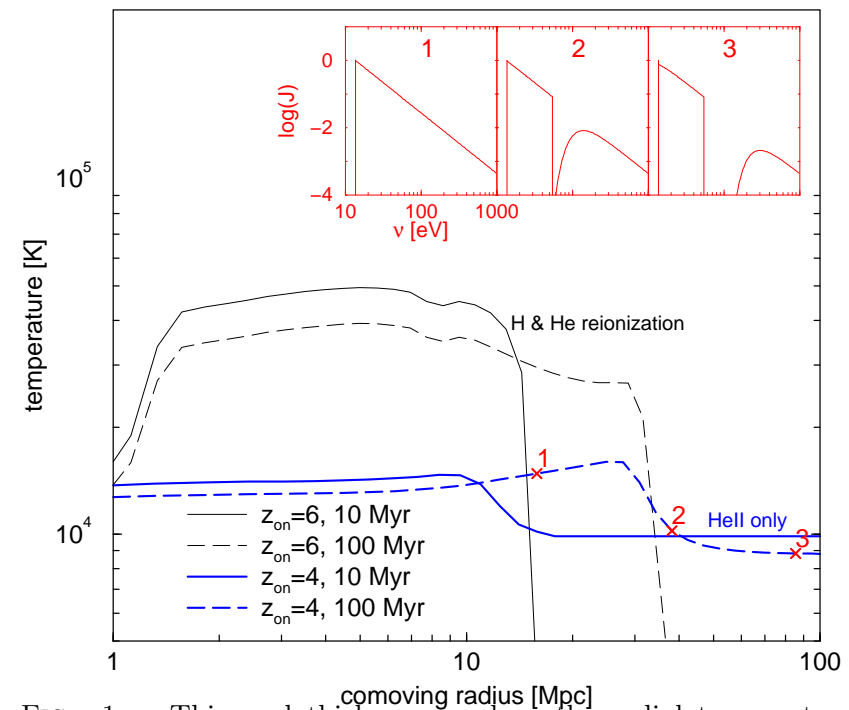

FIG. 1. - Thin and thick curves show the radial temperature profiles of the ionized region around a luminous quasar $\left(N_{\mathrm{ph}}=\right.$ $3 \times 10^{56} \mathrm{~s}^{-1}$ ) that switched on at $z=6$ and $z=4$, respectively. In the first case the ionization front evolves into a completely neutral homogeneous IGM of primordial composition while in the latter case the gas is assumed to be predominatly in the form of H II and HeII . For the second case the inset shows the spectral shape of the ionizing flux at the three different radii marked in the figure.

In the second case only helium is reionized and hydrogen line cooling plays no role due to the small neutral fraction of hydrogen. The mean free path of HeII ionizing photons is about a factor fifty larger than that of hydrogen ionizing photons and the ionization front extends now over a few (comoving) Mpc. The temperature is raised from $9000 \mathrm{~K}$ to about $17000 \mathrm{~K}$ and the total energy input corresponds to $\left\langle E_{\mathrm{ph}}\right\rangle \sim 0.5 \times E_{\mathrm{th}}$, but this value rises to $\left\langle E_{\mathrm{ph}}\right\rangle \sim 0.7 \times E_{\mathrm{th}}$ when the energy deposited ahead of the front is taken into account. The value is again smaller than that in the optical thick limit. This time because the mean free path of an helium ionizing photon becomes larger than the size of the ionized region for $\left(1+E_{\mathrm{ph}} / E_{\mathrm{th}}\right)>3.8(R / 30 \mathrm{Mpc})^{1 / 3}$, where we have assumed that the ionization cross section scales as $\nu^{-3}$ above the threshold. This also explains why the IGM temperature behind the ionization front increases with increasing radius. The inset in Figure 1 shows the spectral shape behind, within and ahead of the front to demonstrate how the spectrum of absorbed photons becomes harder across the ionization front.

\subsection{Photoheating after reonization}

After reionization the mean free path of hydrogen ionizing photons is comparable to the mean distance between hydrogen Lyman limit systems (LLS) which is about $350((1+z) / 4)^{-3}$ Mpc comoving (Storrie-Lombardi 1994).
Much less is known about the number of helium LLS. The model of Miralda-Escudé, Haehnelt, \& Rees (1999, MHR99) which uses a realistic density distribution to treat inhomogeneous reionization predicts a typical distance of $\sim 30 \mathrm{Mpc}$ around $z \sim 3$ (see also Fardal, Giroux \& Shull 1998). Both values are larger than the typical size of numerical simulations and this is is often taken as argument to treat photoheating in the optically thin limit. However, it is not before $\mathrm{z}=1.6$ that the mean free path of hydrogen ionizing photons becomes comparable to the Hubble radius (e.g. Madau, Haardt \& Rees 1999) and the Universe is likely to be opaque to helium ionizing photons up to the present day. Almost all photons emitted at high redshift will therefore be absorbed within a Hubble time and calculations adopting the optically thin limit must severely underestimate at least the mass-weighted average photoheating rate after reionization.

In the case of hydrogen the mean free path of an hydrogen ionizing photon is smaller than the distance between ionizing sources and the optical depth of LLS is large. At a typical place in the Universe the UV background will be dominated by ionizing photons emitted within the region filling the volume to the next LLSs. In the case of HeII the mean distance between sources of ionizing photons at $z \sim 3$ is $\sim 100 \mathrm{Mpc}$ (MHR99) and thus still larger than the mean distance between helium LLS. At a typical point in the Universe the HeII ionizing photons will thereforehave passed a helium LLS. The minimum energy input per ionization is thus raised such that $\left(1+E_{\mathrm{ph}} / E_{\mathrm{th}}\right)>\tau^{1 / 3}$, where $\tau$ is the optical depth at the ionization threshold. Little is known about the optical depth distribution of Helium LLS but even a rather low optical depth of $\tau \sim 10$ would raise the HeII photoheating rate by more than a factor of four compared to the optical thin case.

However, even when the distance between helium LLS falls below the mean distance between sources of ionizing photons the optical thin approximation will significantly underestimate the HeII photo-heating rate, unless helium LLS have a very large optical depth. To understand this consider the extreme case of a homogeneous emissivity of ionizing photons and many helium LLS with optical depth of order unity at the He II ionization threshold. The ionizing background at a given frequency would then be given by the emisssivity times the mean free path at this frequency, $I(\nu) \propto \epsilon(\nu) l(\nu) \propto \epsilon(\nu) \sigma(\nu)^{-1}$. In this limit the mean absorbed photon energy is equal to the mean emitted energy of photons.

An accurate prediction of the mean absorbed energy is extremely difficult as it depends very sensitively on the spatial distribution of HeII optical depths and ionizing sources during and after reionization and on their emitted spectrum. We can here only roughly estimate that $E_{\mathrm{ph}} / E_{\mathrm{th}} \sim 0.5$ to 1 at $z \sim 3$ and should decrease with redshift. The effective HeII photoheating rate should thus be between 2 and 4 times larger than that in the optical thin approximation. It could be even larger, if the emitted spectrum were harder than the $\alpha=1.8$ powerlaw assumed here. 


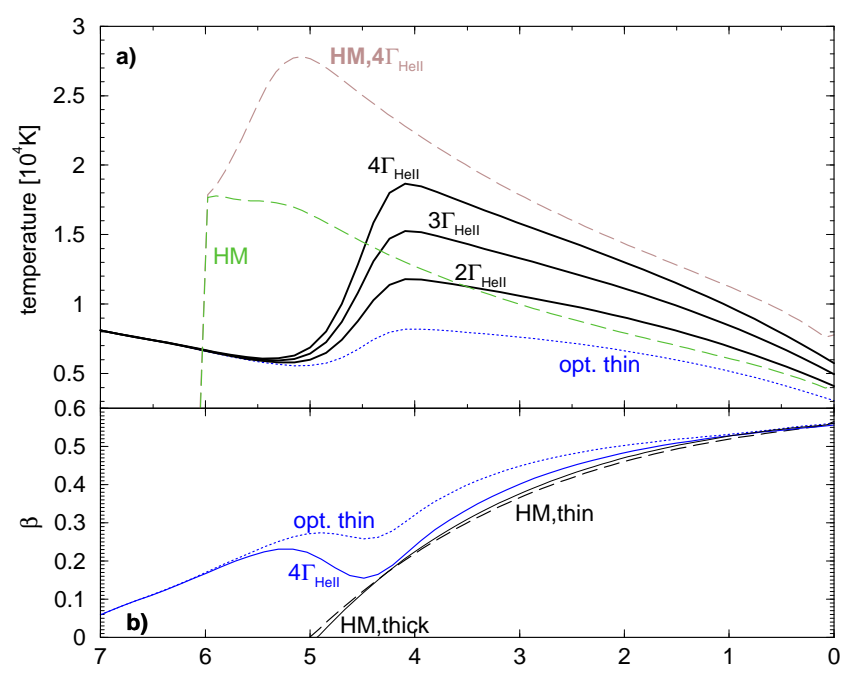

FIG. 2.- a) The temperature evolution of an IGM of primordial composition and mean density assuming a homogeneous UV background as in equation (3). For the solid curves the HeII photoheating rate was raised by a factor $2,3,4$, respectively. The dashed curves assume a homogeneous UV background as given by Haardt \& Madau (1996) with the HeII photoheating for the optical thin limit and raised by a factor four, respectively. b) The slope $\beta=\mathrm{d} \ln T / \mathrm{d} \ln \rho$ of the temperature density relation at mean density.

\section{IMPLICATIONS}

\subsection{Temperature of the IGM}

In the last section we have demonstrated that optical depth effects raise the effective helium photoheating rate by about a factor 2 to 4 . Here we investigate the effect of such an increased helium photoheating rate on the temperature of the IGM. We used a non-equilibrium code to calculate the redshift evolution of the ionization state and temperature of an photoionized IGM of primordial composition with mean baryonic density. We have assumed a homogeneous UV background of the form,

$$
\begin{aligned}
I(\nu, z) & =\left[\frac{1}{1+(7 /(1+z))^{4}} e^{-(z / 4)^{3}}\left(\frac{\nu}{\nu_{\mathrm{HI}}}\right)^{-5}\right. \\
& \left.+\frac{10}{1+(7 /(1+z))^{4}} e^{-(z / 2.5)^{3}}\left(\frac{\nu}{\nu_{\mathrm{HI}}}\right)^{-1.8}\right] \\
& \times 10^{-21} \mathrm{erg} \mathrm{s}^{-1} \mathrm{~Hz}^{-1} \mathrm{sr}^{-1} .
\end{aligned}
$$

This is similar to model C in Haehnelt \& Steinmetz 1998. The first term mimicks reionization of hydrogen by stars before $z=6$. The second component represents the contribution of QSO's to the UV background which here results in a separate epoch of HeII reionization around $z=4$.

The dotted curves shows the temperature evolution for the optical thin case. For the solid curves we have raised the HeII photoheating rate by factors 2,3 and 4 . This corresponds to an increase in the total photoheating rate by a factor 1.3, 2 and 2.6, respectively. For reference the dashed curves show the temperature evolution for the UV background as calculated by Haardt \& Madau (1996) in the optical thin limit and with the HeII photoheating rate increased by a factor of four.

As pointed out by by MHR99 the assumption of an homogeneous UV background during reionization results in an artificially abrupt reionization. In reality reionization should occur over a significant fraction of the Hub- ble time. The assumption of a homogeneous background during reionization should nevertheless give a resonable estimate of the mean temperature after reionization even though there will be considerable spatial temperature fluctuations.

At $z \lesssim 6$ adiabatic cooling exceeds Compton cooling and after helium reionization and in a fully photoionized gas the temperature is thus determined by the balance between the photoheating rate, $\Gamma_{\mathrm{ph}}=\left(\alpha_{\mathrm{HeIII}}\left\langle E_{\mathrm{ph}}^{\mathrm{HeIII}}\right\rangle n_{\mathrm{HeIII}}+\right.$ $\left.\alpha_{\mathrm{HII}}\left\langle E_{\mathrm{ph}}^{\mathrm{HII}}\right\rangle n_{\mathrm{HII}}\right) n_{\mathrm{e}}$ and the adiabatic cooling rate $C_{\mathrm{ad}}=$ $3 k T H(z) \rho / \mu m_{\mathrm{p}}$, where $\alpha\left(\propto T^{-0.7}\right)$ is the recombination coefficient, $n_{e}$ is the electron density, $H(z)$ is the Hubble constant $\mu$ is the mean molecular weight and $m_{\mathrm{p}}$ is the proton mass. Note that there is no explicit dependence on the strength of the radiation field. This results in a quasiequilibrium temperature which is approached at late times (Miralda Escudé \& Rees 1994)

$$
\begin{aligned}
T_{\mathrm{ad}} \sim & 5.9 \times 10^{3}(1+z)^{\frac{1.5}{1.7}}\left(\frac{\rho}{\langle\rho\rangle}\right)^{\frac{1}{1.7}}\left(\frac{\Omega_{\mathrm{b}} h}{0.04}\right)^{\frac{1}{1.7}} \\
& \left(\frac{\left\langle E_{\mathrm{ph}}^{\mathrm{HeIII}}\right\rangle}{E_{\mathrm{th}}^{\mathrm{HeIII}}}+0.15\right)^{\frac{1}{1.7}} \mathrm{~K},
\end{aligned}
$$

where we have taken the optical thin limit for hydrogen heating. The increased HeII photoheating rate due to optical depth effects results in an increase of the temperature by factors $1.5-2.5$ (Fig. 2a). Changing to a cosmological model with $\Omega=0.3$ would further raise this temperature by 20 percent at redshift three because of the decrease in the adiabatic cooling rate.

Madau \& Efsthathiou (1999) recently suggested that Compton heating by the hard X-ray background might significantly contribute to the heating of the IGM. They obtain for the Compton heating rate at the present day $H_{\text {co }}=1.25 \times 10^{-31} \mathrm{erg} \mathrm{s}^{-1}$. We can now compare that to the photoheating rate

$$
\begin{aligned}
\frac{\Gamma_{\mathrm{ph}}}{H_{\mathrm{co}} f(z) n_{e}} & \sim 23\left(\frac{T}{10^{4} \mathrm{~K}}\right)^{-0.7}\left(\frac{\Omega_{\mathrm{bar}} h^{2}}{0.02}\right) \\
& \times\left(\frac{\left\langle E_{\mathrm{ph}}^{\mathrm{HeIII}}\right\rangle}{E_{\mathrm{th}}^{\mathrm{HeIII}}}+0.15\right) \frac{\rho}{\langle\rho\rangle} \frac{(1+z)^{3}}{f(z)} .
\end{aligned}
$$

Note that the photoheating rate incrases with density while the Compton heating rate does not. The redshift evolution of the Compton heating rate $f(z)$ depends on the evolution of the energy density of the hard X-ray background the mean energy of the Compton scattered photon. The Klein-Nishina reduction to the Compton cross section becomes increasingly important at high redshift. Madau \& Eftathiou (1999) obtain $f(z) \sim(1+z)^{13 / 3}$ as approximate redshift dependence if the hard X-ray background was produced at high redshift. Hence, the Compton heating rate by the hard X-ray background exceeds the photo-heating rate only at redshifts larger than

$$
\begin{aligned}
(1+z) & \sim 10\left(\frac{T}{10^{4} \mathrm{~K}}\right)^{-0.52}\left(\frac{\Omega_{\mathrm{bar}} h^{2}}{0.02}\right)^{3 / 4} \\
& \times\left(\frac{\left\langle E_{\mathrm{ph}}^{\mathrm{HeIII}}\right\rangle}{E_{\mathrm{th}}^{\mathrm{HeIII}}}+0.15\right)^{3 / 4}\left(\frac{\rho}{\langle\rho\rangle}\right)^{3 / 4} .
\end{aligned}
$$




\subsection{Absorption properties of the IGM}

The raise in temperature should affect the absorption properties of the IGM in several ways. It will increase the thermal broadening of absorption lines but might also introduce additional non-thermal broadening due to pressure-induced motions. The temperature increase due to optical depth effects during HeII photoheating is therefore a good candidate to resolve recently reported difficulties of numerical simulations to reproduce Doppler parameters of Ly $\alpha$ absorption lines at $z=2$ to 3 as large as those observed (Bryan et al. 1999; Theuns et al. 1999). This would otherwise require an unreasonable large value of $\Omega_{\mathrm{b}} h^{2}$ (Theuns et al 1999). The temperature increase will also increase the mean baryonic density required to match the mean flux decrement in QSO absorption spectra (Rauch et al. 1997). The details of helium heating will furthermore affect the temperature density relation (Hui \& Gnedin 1997) and thus the flux decrement distribution. To demonstrate the effect on the temperature density relation we have plotted in Fig. 2b the logarithmic slope $\beta=\mathrm{d} \ln T / \mathrm{d} \ln \rho$ at mean density for the same models as in Fig $2 \mathrm{a}$.

\subsection{Baryons in shallow potential wells}

The increased helium photoheating rate will also raise the Jeans mass and will decrease the ability of the gas to cool and collapse into shallow potential wells. It will thus affect the overall distribution of baryons. For photoionized gas the dominant cooling processes during the early stages of the collapse are recombination cooling and Bremstrahlung. The equilibrium between photoheating and recombination cooling $\left(C_{\text {rec }} \propto \rho^{2} T^{0.3}\right)$ results in a temperature $T_{\text {rec }} \sim 2.3 \times 10^{5}\left[\left(\left\langle E_{\mathrm{ph}}^{\mathrm{HeIII}}\right\rangle / E_{\mathrm{th}}^{\mathrm{HeIII}}\right)+0.15\right] \mathrm{K}$. The increased HeII photoheating rate should thus affect the circular velocity threshold above which gas can collapse into dark matter haloes and the fraction of gas that can cool in shallow potential wells (Thoul\& Weinberg 1996, ForcadaMiro 1998). Similarly, the increased hydrogen and helium photoheating rates should raise the circular velocity threshold below which baryons in shallow potential wells which collapsed before reionization can be evaporated by an ionization front (Shapiro et al. 1998; Abel \& Mo 1998; Barkana \& Loeb 1999).

\section{CONCLUSIONS}

We have studied radiative transfer effects on the energy input into the IGM due to the reionization and photoheating of hydrogen and helium. The energy input depends crucially on the spectral distribution of the photons actually absorbed.

During reionization the spectrum of an ionizing source becomes harder across the ionization front. The energy input is close to the mean energy of the photons between the ionization threshold and the frequency for which the mean free path of an ionizing photons becomes larger than the radius of the ionized region.

After the reionization of HeII the mean spectrum of ionizing photons above the He III ionization edge will also be considerably harder than the emitted spectrum of an individual source. This is because hard photons can pass through helium LLS. The number and optical depth distribution of helium LLS is still highly uncertain. Thus their effect on the UV specrtum is likely to have been underestimated previously.

The increased HeII photoheating rate due to optical depth effects raises the temperature of the IGM typically by about a factor 1.5 to 2.5 compared to the optical thin approximation. It might thus resolve the problem that current numerical simulations of the Ly $\alpha$ forest (which treat photoheating in the optical thin limit) produce Doppler parameters narrower than observed.

The increased HeII photoheating rate will reduce the ability of the gas to collapse into shallow potential wells. It will also raise the circular velocity threshold below which gas in dark matter haloes is photoevaporated during reionization.

Tom Abel is grateful to Avery Meiksin and Michael Norman for allowing us to show first results from the jointly developed 1-D radiative transfer code. We also thank Greg Bryan, Martin Rees, Tom Theuns and Simon White for stimulating discussions and suggestions. This work was partially supported by NASA through ATP grant NAG54236. Tom Abel acknowledges support from NASA grant NAG5-3923.

\section{REFERENCES}

Abel, T., Anninos, P., Zhang, Y., \& Norman, M. 1997, NewA, 2, 181. Abel, T., Anninos, P., Norman, M.L., \& Zhang, Y. 1998, ApJ, 508, 518.

Abel, T., Meiksin, A., \& Norman, M. 1999, in preperation.

Abel, T., Norman, M.L., Madau, P. 1999, ApJ, in press (astro$\mathrm{ph} / 9812151$ ).

Anninos, P., Zhang, Y., Abel, T., Norman, M.L. 1997, NewA, 2, 209.

Bryan, G.L., Machacek, M., Anninos, P., \& Norman, M.L. 1999, preprint (astro-ph/9805340).

Barkana, R., Loeb, A. 1999, ApJ, submitted, preprint (astro$\mathrm{ph} / 9901114$ ).

Cen, R., Miralda-Escudé, J., Ostriker, J.P., Rauch, M 1994, ApJ, 437, 9L.

Efstathiou, G. 1993, MNRAS, 256, 43

Fardall M.A., Giroux M.L. and Shull J.M., 1999, AJ, 115, 2206.

Forcada-Miro, M.I. 1999, preprint (astro-ph/9712205).

Giroux, M.L., and Shapiro, P.R. 1996, ApJS, 102, 191.

Gunn, J.E., and Peterson, B.A. 1965, ApJ, 142, 1633.

Haardt, F., \& Madau, P. 1996, ApJ, 461, 20.

Haehnelt, M.G., \& Steinmetz, M. 1998, MNRAS, 298, 21L.
Hernquist, L., Katz, N., Weinberg, D.H., \& Miralda-Escudé, J. 1996, ApJ, 457, 51L.

Hui, L., \& Gnedin, N.Y. 1997, MNRAS, 292, 27.

Madau, P., \& Efsthathiou, G. 1999, submitted to ApJ, preprint (astro-ph/9902080).

Madau, P., Haardt, F., \& Rees, M.J. 1999, submitted to ApJ, preprint (astro-ph/9809058).

Miralda-Escudé, J., \& Rees, M.J. 1994, MNRAS, 266, 343.

Miralda-Escudé, J., Haehnelt, M.G., \& Rees, M.J. 1999, submitted to ApJ, preprint (astro-ph/9812306).

Navarro, J.F., \& Steinmetz, M. 1997, ApJ, 478, 13.

Petitjean, P., Müket, J.P., \& Kates, R.E. 1995, A\&A, 295, L9.

Shapiro, P.R., Raga, A.C., \& Mellema, G. 1998, Mem.S.A.It., 69, 463.

Scheuer P., 1965, Nature, 207, 963.

Storrie-Lomardie, L.J, McMahon, R.G., Irwin, M.J., and Hazard, C. 1994, ApJ, 427, L13.

Theuns, T., Leonard, A., Schaye, J., \& Efstathiou, G. 1999, preprint (astro-ph/9812141).

Thoul, A.A. \& Weinberg, D.H. 1996, ApJ, 465, 608.

Zhang, Y. \& Anninos, P., \& Norman, M.L. 1995, ApJ, 453, L57. 